Cahiers

Balkaniques

\section{Cahiers balkaniques}

Hors-série | 2015

Les élites grecques modernes, $\mathrm{XVIII} \mathrm{e}^{\mathrm{X}} \mathrm{XX}^{\mathrm{e}}$ siècles : identités, modes d'action, représentations

\title{
Les évêques orthodoxes et le politique en Thrace grecque à la fin du $\mathrm{xx}^{\mathrm{e}}$ siècle
}

Autorité traditionnelle, rationnelle-légale, charismatique en espace limitrophe et multiconfessionnel

Orthodox Bishops and Politics in Greek Thrace at the End of the 20th Century

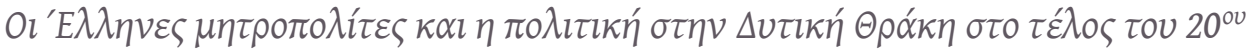

$\alpha \iota \omega ́ v \alpha$

Isabelle Dépret

\section{CpenEdition}

Journals

Édition électronique

URL : https://journals.openedition.org/ceb/5848

DOI : $10.4000 /$ ceb.5848

ISSN : 2261-4184

Éditeur

INALCO

Édition imprimée

Date de publication : 1 mars 2015

ISBN : 978-2-85831-224-5

ISSN : 0290-7402

\section{Référence électronique}

Isabelle Dépret, «Les évêques orthodoxes et le politique en Thrace grecque à la fin du xx $x^{e}$ siècle »,

Cahiers balkaniques [En ligne], Hors-série | 2015, mis en ligne le 17 novembre 2015, consulté le 06

juillet 2021. URL : http://journals.openedition.org/ceb/5848 ; DOI : https://doi.org/10.4000/ceb.5848

Ce document a été généré automatiquement le 6 juillet 2021.

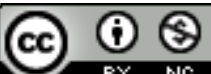

Cahiers balkaniques est mis à disposition selon les termes de la Licence Creative Commons Attribution - Pas d'Utilisation Commerciale 4.0 International. 


\section{Les évêques orthodoxes et le politique en Thrace grecque à la fin du $\mathrm{Xx}^{\mathrm{e}}$ siècle}

Autorité traditionnelle, rationnelle-légale, charismatique en espace limitrophe et multiconfessionnel

Orthodox Bishops and Politics in Greek Thrace at the End of the 20th Century

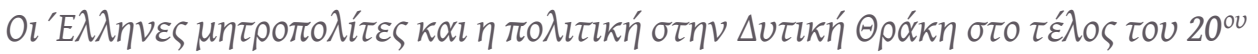
$\alpha \iota \omega ́ v \alpha$

Isabelle Dépret

\section{Introduction}

1 Fin mars 2013, peu après l'intronisation, à Komotini, d'un nouvel évêque orthodoxe, la presse hellénophone de Thrace commentait les visites réciproques «à fort contenu symbolique » entre le nouveau métropolite et le chef religieux musulman local. Reçu au siège de la métropole, le mufti offrait à son hôte la copie d'un traité daté de 636 , conclu entre le calife et le Patriarche grec orthodoxe. Pour lui, ce texte illustrerait avec force les principes de respect entre confessions et de collaboration entre dirigeants religieux. En réponse, l'évêque $\mathrm{M}^{\mathrm{gr}}$ Pandeleïmon Moutafis appelait les «autres peuples » à s'inspirer « des relations exemplaires, en Thrace, entre musulmans et chrétiens ainsi qu'entre leurs chefs religieux respectifs ${ }^{1}$. Ces gestes, ces déclarations, reflets d'un contexte favorable en 2013, invitent à s'interroger sur la place des élites religieuses dans l'État grec, notamment en contexte frontalier.

La Thrace - aux confins de la Turquie et de la Bulgarie - présente une configuration bien particulière au $\mathrm{xx}^{\mathrm{e}}$ siècle $^{2}$. Dans un État-nation majoritairement chrétien ${ }^{3}$, cette région située au Nord-Est du pays est restée ouvertement pluriconfessionnelle, multiculturelle. En 1923, les musulmans de la région ont, en effet, été exemptés des échanges de populations conclus entre la Grèce et la Turquie ${ }^{4}$. Depuis lors, ces citoyens 
Grecs musulmans, qu'ils soient d'origine turque, slave ou rom, relèvent, aux yeux de

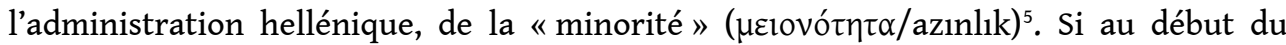
$\mathrm{xx}^{\mathrm{e}}$ siècle, la Thrace occidentale abritait de petites communautés arméniennes et juives $^{6}$, à la fin du siècle, les chrétiens orthodoxes constituent, face aux musulmans, l'autre communauté confessionnelle majeure.

3 Si l'on reprend les catégories wébériennes ${ }^{7}$, les évêques orthodoxes représenteraient $a$ priori, en Grèce, des élites «traditionnelles » : ils occupent en effet un rang supérieur, une position de pouvoir à l'échelle locale et étatique ${ }^{8}$ et leur autorité s'appuie sur la force de la tradition ${ }^{9}$. Les actes, les paroles de ces dirigeants se réfèrent constamment à l'impératif de continuité entre le passé et le présent ${ }^{10}$.

4 En me centrant donc sur la Thrace occidentale au $\mathrm{xx}^{\mathrm{e}}$ siècle, sur les évêques et leurs rapports à l'enjeu minoritaire/musulman, je me demanderai jusqu'à quel point ces hauts responsables peuvent être envisagés comme des élites traditionnelles. Je m'interrogerai par ailleurs sur le type de rapports entretenus avec d'autres autorités nationales et sur l'évolution possible de leur rôle au début du xxie siècle.

\section{Élites religieuses en Thrace grecque : contraintes et ressources d'une configuration limitrophe et multiconfessionnelle}

\section{Un espace multiconfessionnel dans un État majoritairement orthodoxe}

5 En dépit d'un mouvement de sécularisation, accéléré à la fin du xxe siècle, l'État grec ne s'est jamais posé comme laïc, au sens strict: l'Église orthodoxe y jouit, depuis le $\mathrm{XIX}^{\mathrm{e}}$ siècle, d'une position institutionnelle, juridique prédominante ${ }^{11}$. Les conditions d'émergence de l'État - par émancipation du cadre ottoman - ont favorisé le maintien d'un lien étroit entre nationalité grecque et christianisme orthodoxe. Après l'établissement d'un royaume indépendant en 1830, l'islam et la turcité, associés à un Empire rejeté, ont largement été tenus pour étrangers ${ }^{12}$. Jusqu'au milieu du $\mathrm{xx}^{\mathrm{e}}$ siècle, de même qu'en Turquie voisine -, la construction étatique s'est accompagnée d'une certaine mise à l'écart, parfois de l'expulsion de l'altérité religieuse ${ }^{13}$.

Ottomane jusqu'aux guerres balkaniques, la Thrace occidentale ${ }^{14}$ est d'abord attribuée à la Bulgarie ${ }^{15}$. Ce n'est qu'à la fin de la Première Guerre mondiale (Traité de Sèvres du 28 juillet 1920) que la région est rattachée à l'État grec ${ }^{16}$. L'armée française, qui supervise la région en 1919-1920, présente dans ses rapports un espace pluriconfessionnel majoritairement musulman, essentiellement agricole, structuré autour d'une plaine marécageuse malsaine ${ }^{17}$.

7 Le passage de l'Empire à l'État nation s'est traduit par une évolution des rapports de force socioconfessionnels. En effet, au début du $\mathrm{xx}^{\mathrm{e}}$ siècle, les musulmans contrôlent plus de $80 \%$ de la terre ${ }^{18}$. Cette proportion se réduit dès l'entre-deux-guerres. Y contribuent l'arrivée d'environ 150000 réfugiés chrétiens venus de Thrace orientale et d'Anatolie ainsi que plusieurs réformes agraires, qui démantèlent la grande et moyenne propriété foncière ${ }^{19}$. Une politique d'hellénisation - démographique, toponymique - a également été menée ${ }^{20}$ : à la fin des années 1920 , selon les autorités grecques, les 
musulmans seraient environ 100000 pour un total de 300000 personnes dans la région ${ }^{21}$.

Citoyens grecs, les musulmans de Thrace jouissent pourtant, conformément au Traité de Lausanne (1923), de droits collectifs: fonctionnement d'écoles primaires et secondaires "minoritaires ", où les cours sont dispensés en grec et en turc; maintien de la juridiction du mufti, qui conserve après 1923 des compétences en droit familial. Dans la seconde moitié du $\mathrm{xx}^{\mathrm{e}}$ siècle, 3 muftis officiels, nommés et rémunérés par l'État sont en fonction en Thrace (à Xanthi, Komotini et Didymoteicho/Alexandroupolis). Enfin, est reconnue, sous contrôle étatique, l'autogestion des wakfs, ces biens communautaires issus des fondations pieuses ottomanes ${ }^{22}$. À la fin $d u x^{e}$ siècle, la Thrace grecque compte plus de 300 mosquées, quelques tekke, 235 écoles et 3 lycées minoritaires, plusieurs écoles coraniques ${ }^{23}$.

Jusqu'aux années 1990 au moins, les musulmans ont été volontiers perçus par l'État grec comme des populations quasi étrangères, inassimilables. Ces populations sont aussi apparues comme contrepoids de la minorité grecque orthodoxe d'Istanbul, une communauté plutôt malmenée en Turquie au $\mathrm{xx}^{\mathrm{e}}$ siècle ${ }^{24}$. Cette logique de « réciprocité », souvent mentionnée, a biaisé les relations entre le pouvoir et les résidents musulmans, de même que les rapports interconfessionnels sur le terrain. Cette réciprocité n'impliquait-elle pas, en effet, que toute mesure offensive prise par l'État turc - a fortiori envers les Rums d'Istanbul - devait automatiquement impliquer des représailles à l'encontre des musulmans de Thrace ? Ce regard et ces pratiques ont été reconnus au sommet de l'État au début des années 1990 tandis qu'une autre manière d'envisager ces citoyens musulmans est alors préconisée ${ }^{25}$. Cette manière longtemps diffuse d'envisager l'autre confessionnel comme un outil de relations internationales plus que comme un individu ou un citoyen a été pointée après la fin des années $1990^{26}$.

\section{L'Église orthodoxe en Thrace, un acteur incontournable à l'échelle locale}

10 Dans un contexte marqué par le poids des enjeux sécuritaires et diplomatiques, l'Église orthodoxe a conservé un rôle social important à l'échelle locale. Ainsi, après 1923, la communauté confessionnelle, l'église locale auraient constitué, pour les réfugiés chrétiens, un canal d'intégration à l'État grec, un moyen de recréer des liens, des racines : au début des années 1990, dans la région de Komotini, la grande majorité des orthodoxes serait issue de familles réfugiées réinstallées en Grèce au début des années $1920^{27}$. Face aux mosquées, les lieux de culte chrétiens - églises, chapelles, monastères - marquent aussi l'ancrage dans le territoire d'une communauté.

11 Plus qu'à Athènes, la vie religieuse et la piété populaire se sont maintenues au $\mathrm{xx}^{\mathrm{e}}$ siècle dans cet espace resté fortement rural: ainsi, les fêtes patronales, la vénération des saints, les pèlerinages sont l'occasion de vastes rassemblements systématiquement couverts par la presse locale. L'Église orthodoxe a assuré dans ce cadre, un rôle important de garant de la coexistence interconfessionnelle, mais aussi de pôle identitaire ${ }^{28}$. L'activité et l'empreinte personnelle des élites ecclésiastiques doivent être replacées dans cette configuration, qui a représenté autant de contraintes que de possibles ressources. 
12 En Thrace grecque, l'Église orthodoxe est structurée en 4 diocèses: Maroneia/ Komotini ; Xanthi/Peritheoriou ; Alexandroupolis ; Didymoteicho/Orestiada ${ }^{29}$. Les diocèses de Xanthi et de Komotini s'inscrivent dans un environnement à forte proportion musulmane. Toutefois, dans les deux autres évêchés - proches des frontières bulgares et turques -, la thématique sécuritaire est restée prégnante ${ }^{30}$.

Les relations entre chrétiens et musulmans au xxe siècle, mêlées de subtiles méfiances interconfessionnelles ${ }^{31}$, ont été le plus souvent pacifiques - un aspect valorisé par nombre d'acteurs politiques au tournant des $\mathrm{xx}^{\mathrm{e}}$ et $\mathrm{xxI}^{\mathrm{e}}$ siècles. À l'échelle des autorités religieuses, les protections du Traité de Lausanne ont consolidé un respect des espaces de juridictions ${ }^{32}$ d'autant que le prosélytisme a toujours été un délit condamné par la Constitution $^{33}$.

\section{Des élites traditionnelles ? Formation, parcours d'évêques orthodoxes en deuxième partie du $x x^{\mathrm{e}}$ siècle}

\section{Une légitimation rationnelle-légale}

Depuis 1923, l'élection des évêques ressort des organes ecclésiastiques ${ }^{34}$. Selon la Charte de 1977, les métropolites sont choisis par l'Assemblée des évêques. Les candidats éligibles sont des prêtres célibataires à la "foi droite ", aux mœurs "irréprochables ", âgés d'au moins 35 ans, diplômés d'une Université depuis cinq ans au moins, dotés d'une expérience pastorale appréciée et de nationalité grecque ${ }^{35}$.

L'autorité des évêques orthodoxes en Grèce renvoie donc à une double sanction. La première, - la plus décisive-, est celle de l'Église, plus exactement de l'élite sacerdotale, les 80 métropolites orthodoxes du pays. Cette élection est le couronnement d'un parcours professionnel dans les structures ecclésiastiques. La deuxième sanction est celle de l'État, tenu de reconnaître une élection régulière en publiant un décret présidentiel de nomination. Les évêques orthodoxes, assimilés à de hauts fonctionnaires, sont dès lors salariés de l'État ${ }^{36}$.

Cette double sanction indique que l'autorité de l'évêque orthodoxe en Grèce est donc aussi fondée sur des critères "rationnels-légaux ", pour reprendre le vocabulaire de Max Weber ${ }^{37}$. Les évêques doivent avoir effectué des études de théologie, être titulaires d'un diplôme académique réalisé le plus souvent dans les Universités d'État, à Athènes ou à Salonique. Ils doivent par ailleurs avoir franchi les échelons de la hiérarchie et disposer d'une solide expérience.

Sur cette période chronologique, la majorité des évêques de Thrace a plutôt emprunté une carrière locale, ancrage favorisé par l'institution ${ }^{38}$. C'est le cas de Damaskinos Rouméliotis, évêque de Maroneia et Komotini de 1974 à 2012 : né en 1920 dans un village du Péloponnèse - et après s'être "distingué » dans l'armée hellénique durant la Seconde Guerre mondiale et la Guerre civile -, il s'installe en 1958 à Komotini, à l'invitation du métropolite d'alors. Il y exerce pendant 16 ans comme prêtre prédicateur avant d'être élu métropolite du diocèse, lorsque l'évêque Timotheos est muté à Néa Ionia, en Attique ${ }^{39}$. $\mathrm{M}^{\mathrm{gr}} \mathrm{Nikiforos}$, l'évêque de Didymoteicho depuis novembre 1988, a aussi emprunté cette voie locale : né à Ferrès, dans le département de l'Evros - en Thrace donc -, il exerce 14 ans la fonction de Protosynkelos de la métropole d'Alexandroupolis, dans l'ombre de l'influent évêque local, $\mathrm{M}^{\mathrm{gr}}$ Anthimos. L'actuel 
évêque de Komotini, $\mathrm{M}^{\mathrm{gr}}$ Pandeleimon Moutafis, a été Secrétaire général de la métropole de Xanthi pendant plus de 25 ans ${ }^{40}$. élus et ordonnés sont en effet considérés comme les successeurs des Apôtres et seraient, comme eux, inspirés par le Saint-Esprit ${ }^{43}$. Cet ascendant peut être consolidé par la personnalité de l'évêque, ses relations avec les fidèles, son éloquence. À cet égard, les évêques d'Alexandroupolis, Anthimos, et de Maroneia, Damaskinos, - en exercice en Thrace au cours des dernières décennies du $\mathrm{xx}^{\mathrm{e}}$ siècle - ont pu être tenus pour des élites charismatiques et, en tout cas, visibles et médiatiques ${ }^{44}$.

21 L'origine sociale des évêques de la région au $\mathrm{xx}^{\mathrm{e}}$ siècle relève de la petite surtout, ou de la moyenne bourgeoisie. L'évêque Anthimos d'Alexandroupolis a eu une mère institutrice, un père propriétaire et agriculteur, mais la famille élargie compte des membres du Parlement. $\mathrm{M}^{\mathrm{gr}}$ Damaskinos de Komotini semble, en revanche, provenir d'un milieu rural modeste : il aurait travaillé comme employé de commerce aussitôt après l'école primaire avant de reprendre ultérieurement des études ${ }^{45}$. L'entrée dans une carrière sacerdotale au sein de l'Église de Grèce a-t-elle pu représenter au xx siècle un mode d'ascension et de reconnaissance sociales?

Enfin, l'attitude, l'argumentation des évêques ont été influencées par un environnement auquel il a fallu s'ajuster. L'examen d'une période critique met en relief cet aspect.

\section{Tensions intercommunautaires et élites ecclésiastiques à la fin du $x x^{\mathrm{e}}$ siècle}

\section{Une période critique}

Pour ce qui concerne la gestion par l'État de la «minorité », la place des musulmans dans la société locale, la qualité des rapports interconfessionnels, les années 1980-1990 constituent une période charnière en Thrace. 
Les années 1980 correspondent en effet en Grèce à une consolidation des institutions démocratiques, mais aussi à une revitalisation du discours de fierté et d'indépendance nationales. Ces tendances ont d'abord peu profité aux «minorités » - linguistiques, culturelles, religieuses - considérées comme des menaces, dans un climat de bras de fer gréco-turc. Paradoxalement, la politique de méfiance ou d'endiguement de la présence musulmane en Thrace, formulée dans les années 1960, se maintient dans les années $1980^{46}$. Ce type de gestion ne sera remis en cause au sommet de l'État qu'à partir des années 1990. Durant ces années, la logique ancienne de nationalisation des appartenances confessionnelles ${ }^{47}$ prend un tour violent en 1990 à Komotini ${ }^{48}$.

Les élites religieuses orthodoxes n'ont pas répondu de la même manière à ce défi et aux attentes - d'ailleurs contradictoires - des fidèles et des milieux politiques. Au cours des trois dernières décennies $d u x^{e}$ siècle, dans leurs rapports à l'enjeu minoritaire et à la pluralité confessionnelle, deux profils de métropolites tendent à émerger.

\section{Évêques orthodoxes et politique}

6 D'une part, nous retrouvons des évêques centrés sur des fonctions spirituelles, liturgiques, sociales et administratives, plutôt considérés comme des «traits d'union » ou des "pacificateurs ": les métropolites de Xanthi dans la seconde moitié du $\mathrm{xx}^{\mathrm{e}}$ siècle se rattacheraient plutôt à ce modèle, dans un contexte culturel marqué, dans le Nord de la circonscription, par une multiplicité de villages musulmans d'origine pomaque ${ }^{49}$. Au moment des tensions intercommunautaires des années 1980-1990, les déclarations et actions de l'évêque de Xanthi auraient joué dans le sens de l'apaisement. Au début des années 2000, l'évêque Pandeleïmon Kalafatis joue un rôle clé dans l'édition d'un ouvrage consacré aux monuments religieux de la préfecture de Xanthi en collaboration avec l'administration du mufti local. Cet ouvrage, réalisé avec l'appui des autorités gouvernementales, est emblématique dans la mesure où il accorde de manière assez inédite aux anciens bâtiments ottomans et lieux de culte musulmans un intérêt, une valeur au même titre que les espaces sacrés chrétiens ${ }^{50}$. Le nouvel évêque de Komotini, Pandeleïmon Moutafis, ancien cadre de l'évêché de Xanthi rejoindrait cette logique.

Le second profil idéal typique est celui d'évêques qui, en tant que chefs de l'Église orthodoxe, se sont posés en pôles de combat en faveur d'une communauté ethnoreligieuse. Dans ce cas, la pluralité confessionnelle n'est pas véritablement acceptée, l'altérité est perçue comme dangereuse, illégitime.

Les évêques d'Alexandroupolis, Anthimos, (1974-2004) et de Maroneia, Damaskinos (1974-2012), entrent parfaitement dans cette catégorie. Ces deux évêques, nés dans l'entre-deux-guerres, ont ainsi combiné un travail pastoral, philanthropique à une mission en faveur de l'hellénisme menacé. Pour ces hommes d'Église, la menace est d'abord celle d'une «submersion de l'élément chrétien » par des familles musulmanes plus nombreuses. À l'instar d'autres acteurs locaux, $\mathrm{M}^{\mathrm{gr}}$ Anthimos et $\mathrm{M}^{\mathrm{gr}}$ Damaskinos dénoncent avec virulence les stratégies expansionnistes de la Turquie dans la région: les thèmes de la menace turco-islamique et de l'Église luttant aux frontières pour la Nation sont alors abondamment repris dans les médias, en chaire ou encore au sein des instances ecclésiastiques centrales ${ }^{51}$. Les deux hauts responsables mettent aussi à leur actif d'avoir fait édifier nombre de nouvelles églises dans leur diocèse - plus de 30 dans le diocèse d'Alexandroupolis, une quinzaine dans celui de Maroneia -, et d'avoir chacun 
inauguré un musée ecclésiastique, avec l'appui financier des ministères de Macédoine orientale-Thrace et de la Culture.

L'évêque représente un notable incontournable dans son diocèse. Ses rapports aux autres élites locales (administratives, judiciaires, politiques, associatives, académiques, éducatives, militaires et médiatiques) sont le plus souvent de l'ordre de la reconnaissance et de la collaboration, au moins formellement.

Les deux évêques précités ont quant à eux utilisé leur autorité, les liens institutionnels entre l'Église orthodoxe et l'État, mais aussi cette référence aux «questions nationales ", pour façonner leurs relations aux fidèles, aux autres acteurs régionaux. Cet aspect apparait dans les nombreux articles de la presse locale évoquant ces personnalités religieuses ainsi que dans un volume dédié à $\mathrm{M}^{\mathrm{gr}}$ Damaskinos, publié en 2005. Dans cet ouvrage collectif, des autorités ecclésiastiques, administratives, politiques, policières, militaires, académiques apportent leur contribution à l'évêque de Maroneia $^{52}$. Ici, les liens avec les d'autres membres du haut clergé - dont le Patriarche de Constantinople, $\mathrm{M}^{\mathrm{gr}}$ Bartholomaios - ont aussi compté. Ce n'est pas un hasard si en 1999, le Synode de l'Église de Grèce, à l'initiative de l'archevêque d'Athènes, décide de soutenir financièrement les familles nombreuses orthodoxes de Thrace, avec en toile de fond ce " combat » des évêques locaux et ce danger turco-musulman ${ }^{53}$.

Les relations des évêques avec les autres élites locales ont-elles été toujours été de l'ordre de la complicité? Non, bien sûr et si l'on reprend le cas des métropolites Anthimos et Damaskinos, leur forte visibilité, leur engagement dans l'espace public, leurs discours nationalistes, leur ont valu autant de soutiens, de popularité que de critiques. Après 1974, outre les membres de la minorité musulmane, une partie des citoyens, des élus, des intellectuels, des fonctionnaires ont dénoncé la tendance de ces deux évêques à envenimer les relations interreligieuses et surtout, à empiéter sur les compétences d'autres acteurs légitimes: hommes politiques, administration civile, syndicats ou encore justice. Ainsi, à Komotini, les relations entre l'évêque et le conseil municipal sont plutôt tendues au cours des années 1990, d'autant que certains conseillers sont musulmans.

\section{Conclusion}

32 À la fin du $\mathrm{xx}^{\mathrm{e}}$ siècle, les membres du haut clergé orthodoxe ont le plus souvent associé plusieurs formes d'autorité et de registres. La tradition ne représente donc pour ces « élites » qu'une forme, certes cruciale, de leur légitimation.

33 Si en Grèce contemporaine, les évêques orthodoxes entretiennent le plus souvent des relations de courtoisie, de coopération avec les dirigeants locaux ou nationaux ${ }^{54}$, leurs contacts avec les milieux intellectuels, artistiques ou politiques n'ont pas exclu pressions réciproques ou frictions : ce fut le cas à Komotini ou à Alexandroupolis à la fin du xxe siècle. Quant aux rapports avec l'Autre confessionnel, ils ont le plus souvent été peu développés au $\mathrm{xx}^{\mathrm{e}}$ siècle, empreints de suspicion, biaisés par des facteurs politiques.

Dans un ouvrage célèbre, Charles Wright Mills considérait qu'aux États Unis les élites politiques, économiques et militaires ne constituaient, au milieu $d u x^{e}$ siècle, qu'un seul bloc solidaire ${ }^{55}$. Les rapports entre groupes de pouvoir ont parfois été plus complexes. L'exemple de la Thrace grecque à la fin du $\mathrm{xx}^{\mathrm{e}}$ siècle en atteste, dans un 
pays pourtant où les relations entre Église et État sont le plus souvent posées en termes d'harmonie.

\section{BIBLIOGRAPHIE}

AKGÖNÜL Samin, 1999, Une communauté, deux États, Cahiers du CERATO, Istanbul : Isis.

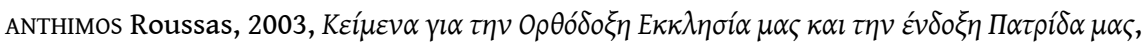
Alexandroupolis : Gnorimia.

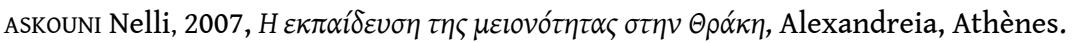

BIYıLıoĞLU Tevfik, 1955, Trakya'da millî mücadele, Ankara : Türk Tarih Kurumu Basımevi.

DALÈGRE Joëlle, 1997, la Thrace grecque. Populations et territoire, L'Harmattan, Paris.

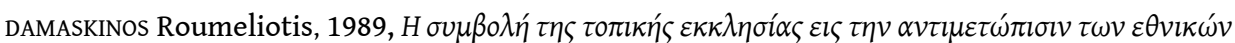
$\pi \rho \circ \beta \lambda \eta \mu \alpha ́ \tau \omega \nu \tau \eta \varsigma \Theta \rho \alpha ́ \kappa \eta \varsigma$, éditions de la Sainte Métropole de Komotini, Komotini.

DÉPRET Isabelle, 2009, Église orthodoxe et histoire en Grèce contemporaine. Versions officielles et controverses historiographiques, L'Harmattan, Paris.

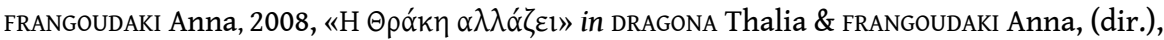

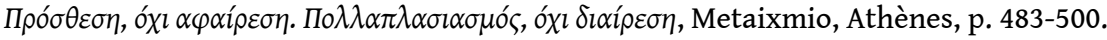

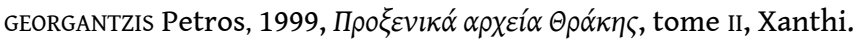

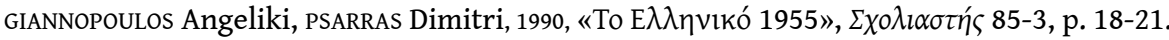

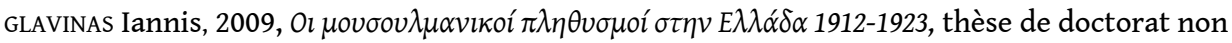
publiée, Histoire moderne et contemporaine, Université Aristote de Salonique.

HERSANT Jeanne, 2008, « Surveillances croisées et rivalités gréco-turque en Thrace occidentale », European Journal of Turkish Studies 8, URL : http://ejts.revues.org/2693.

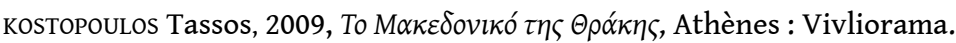

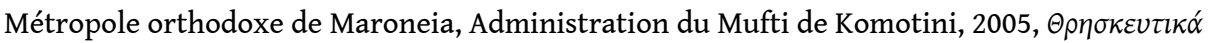

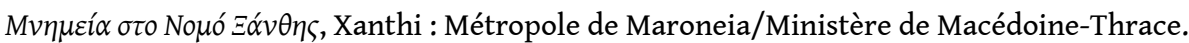

PAPAZOGLOU Georgios, (dir.), 2006, A $\Delta \alpha \mu \alpha \sigma \kappa \eta v o ́$, Komotini : Thrakiki Vivliothiki.

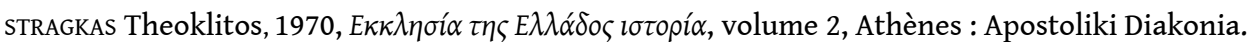

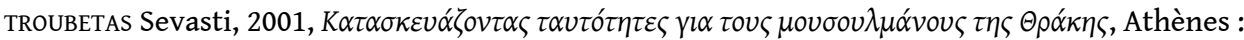
Kritiki/KEMO.

TSITSELIKIS Konstantinos, 2012, Old and New Islam in Greece, Leiden: Martinus Nijhoff Publishers.

TSITSELIKIS Konstantinos, 2007, “The Pending Modernisation of Islam in Greece”, Südosteuropa 55-4, p. 354-372.

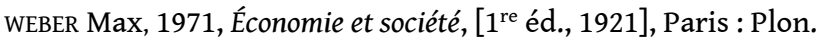




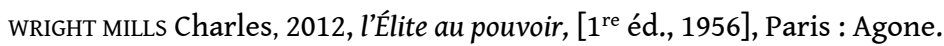

\section{NOTES}

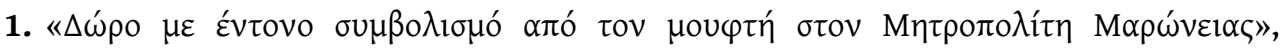

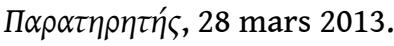

2. DALÈGRE J., 1997, la Thrace grecque, L'Harmattan, Paris ; TSITSELIKIS K., 2012, Old and New Islam in Greece, M. Nijhoff, Leiden.

3. FRANGOUDAKI A., DRAGONA Th. (dir.), 1997, Tív $\alpha \imath \eta \Pi \alpha \tau \rho i ́ \delta \alpha \mu \alpha \varsigma$, Alexandreia, Athènes.

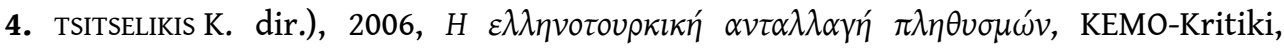
Athènes.

5. Ministère des Affaires étrangères hellénique, “The Muslim Minority of Greek Thrace", http://www.hri.org/MFA/foreign/musminen.htm, consulté le 27 octobre 2015 ; AKGÖNÜL S., 1999, Une communauté, deux États, Cahiers du CERATO/ISIS,

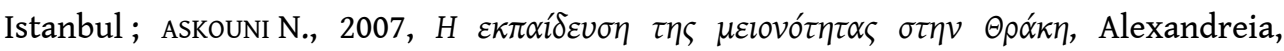
Athènes.

6. Les recensements de 1906 et de 1912 soulignent l'importance numérique des Bulgares exarchistes en Thrace: données reproduites dans KosTopoulos T., 2007,

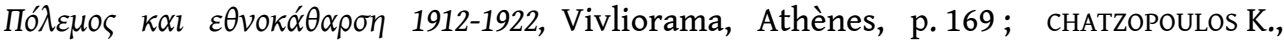

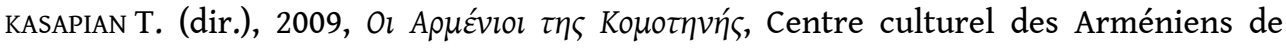
Komotini, Komotini.

7. WEBER M., 1971 (1921), Économie et société, chapitre 3, Plon, Paris.

8. Le terme d'élite inclut une connotation normative, puisqu'il évoque à la fois les " meilleurs » et ceux qui ont été « choisis ». Au sens courant, il désigne un groupe social situé au sommet de la hiérarchie, détenant du pouvoir. CHARLE C., "Éléments pour une histoire comparée des élites en France et en Europe occidentale ( $\mathrm{XIX}^{\mathrm{e}}-\mathrm{XX}^{\mathrm{e}}$ siècles) ", Actes de la recherche en sciences sociales 116-117 (1997), p. 39-52.

9. Pour un regard interne, WARE T., 1997, L'Orthodoxie, Desclée de Brouwer, Paris.

10. HERVIEU-LÉGER D., «Tradition, traditionalisme et néo-traditionalisme », in AZRIA R., HERVIEU-LÉGER D. (dir.), 2010, Dictionnaire critique des faits religieux, PUF, Paris, p. 539-540.

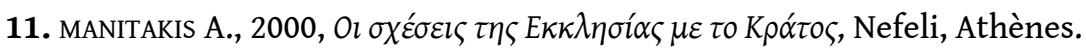

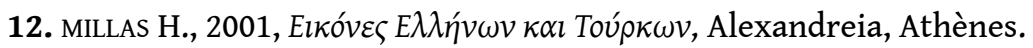

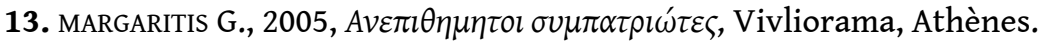

14. BIYILIOĞLU T., 1955, Trakya'da millî mücadele, Türk Tarih Kurumu Basımevi, 1955.

15. DRIAUlT E., LHÉRITIER M., 1925, Histoire diplomatique de la Grèce de 1921 à nos jours, tome 4, PUF, Paris, p. 92-94.

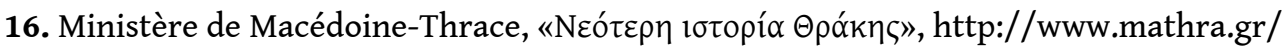
default_270.aspx, consulté le 27 octobre 2015.

17. AMAEG, 1921/02, dossier 4, 8, 1; 7, 1, 1 et 7, 2, 2 .

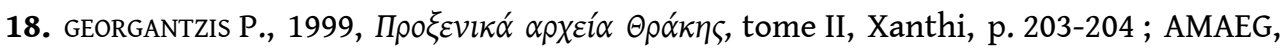

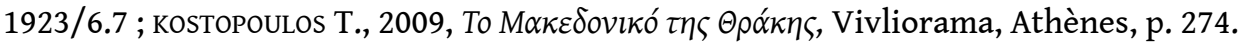


19. PÉCHOUX P.-Y., 1975, « La réforme agraire en Grèce ", Revue géographique de Lyon 50-4,

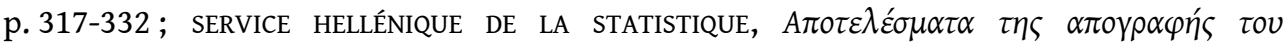

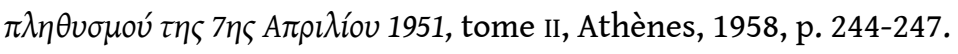

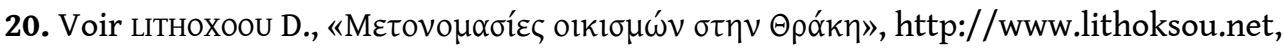
consulté le 27 octobre 2015.

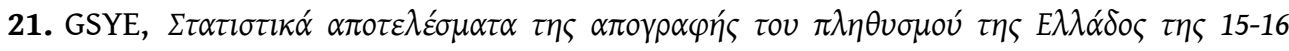
Maíov 1928, volume IV, Athènes, p. $\kappa \gamma-\rho \beta-\rho \gamma$.

22. Le wakf est en droit islamique, une donation à une œuvre d'utilité publique, philanthropique ou religieuse: mosquées, écoles religieuses, institutions sociales, hôpitaux, mais aussi terres, magasins, appartements loués.

23. Ministère des Affaires étrangères hellénique, art. cit. TSITSELIKIS K., KURBAN D., 2010, A Tale of Reciprocity, Tesev/Kemo, Istanbul/Athènes.

24. DILEK Güven, «6-7 Eÿlül Olaylari», Radikal, 9 juin 2006 ; VRYONIS S., 2005, The Mechanism of Catastrophe, The Turkish Pogrom of 6-7 September 1955, Greekworks, New York.

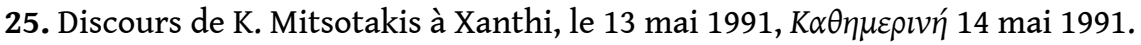

26. TSITSELIKIS K., Old and New..., op. cit., p. 105-117 ; HERSANT J., « Surveillances croisées et rivalités gréco-turque en Thrace occidentale ", European Journal of Turkish Studies 8 (2008), URL : http://ejts.revues.org/2693, consulté le 27 octobre 2015.

27. Témoignages de cadres de la métropole orthodoxe de Komotini, d'habitants de Komotini âgés de plus de 70 ans, étude de terrain en Thrace grecque, mai 2010, juillet 2011.

28. Idem.

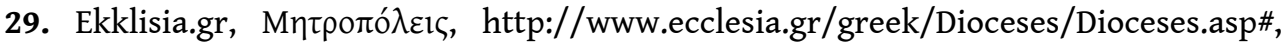
consulté le 27 octobre 2015 ; Saint Synode de l'Église de Grèce, 1919, 1921, $\Sigma v \lambda \lambda o \gamma \eta ́$

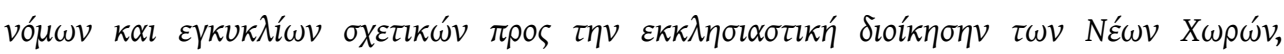
2 volumes, Apostoliki Diakonia, Salonique.

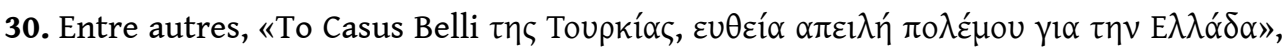

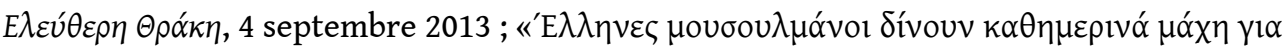

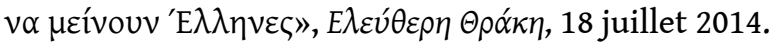

31. Témoignages de chrétiens et de musulmans à Xanthi, Alexandroupolis, Komotini, dans plusieurs villages du Nord de l'Evros, mai 2010, juillet 2011.

32. La mise en question de l'appartenance pleine des Bektachis/Alévis ou des Pomaques à la communauté musulmane, projet plus récent, serait davantage le fait d'hommes politiques, d'intellectuels que du haut clergé. DÉPRET I., 2014, à paraître, «Islam hétérodoxe et christianisme en Thrace. Tabous, identités religieuses et discours nationaux ", Archives de sciences sociales des religions; TROUBETAS S., 2001,

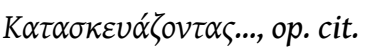

33. Constitution hellénique de 1975, article 13.

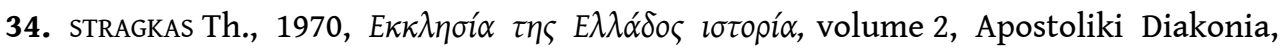
Athènes, p. 1265-1266.

35. Charte statutaire de l'Église de Grèce (1977), articles 17 à 28.

36. DÉPRET I., Église orthodoxe et histoire, op. cit., p. 133-138. 
37. WEBER M., «La domination légale à direction bureaucratique », in TESSIER R. (dir.), 1991, Théories de l'organisation, Presses universitaires de Québec, Montréal, p. 23-32.

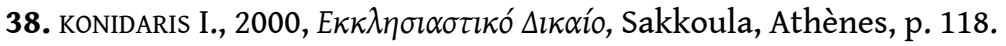

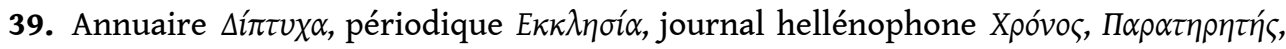

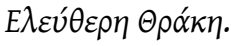

40. Idem.

41. Idem.

42. WEBER M., 1971 (1921), Économie et société, chapitre 3, Plon, Paris.

43. KONIDARIS I., 2000, op. cit., p. 118-119

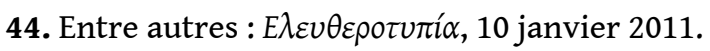

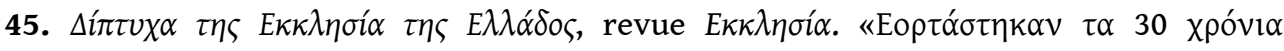

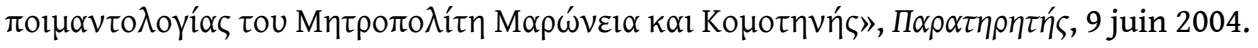

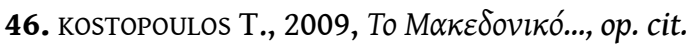

47. Le juriste et historien K. TSITSELIKIS parle de "néo-millet ", "The Pending Modernisation of Islam in Greece”, Südosteuropa 55-4 (2007), p. 354-372.

48. En janvier 1990, une altercation grave entre un musulman et un chrétien à l'hôpital de Komotini, l'emprisonnement du député musulman indépendant Ahmet Sadik, une manifestation de musulmans revendiquant le droit de se déclarer « Turcs » cristallisent une flambée de violence le 29 janvier 1990. Celle-ci est menée par des «chrétiens indignés » contre des maisons et des boutiques musulmanes. Cet événement, qualifié de "pogrom » par la presse turque, a représenté un électrochoc pour la classe politique grecque. Pour un point de vue critique: GIANNOPOULOS A., PSARRAS D., 1990, «To

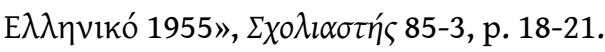

49. Antonios Klaudatos, évêque de Xanthi de 1954 à 1994 a exercé durant l'entre-deuxguerres à Florina, où résident alors nombre de slavophones.

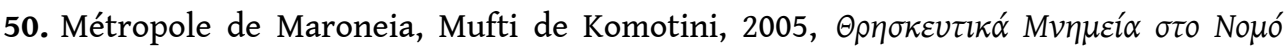
$\Xi \alpha ́ v \theta \eta \varsigma$, édition bilingue gréco-turque financée par la préfecture de Macédoine orientale-Thrace et l'Union européenne, Xanthi.

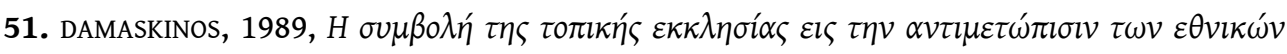

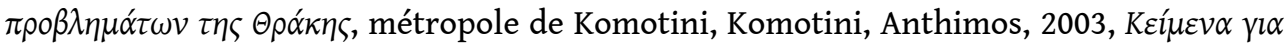

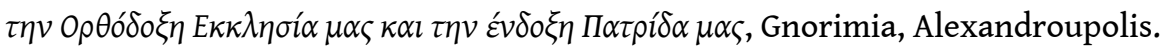

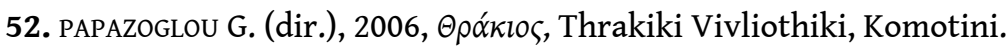

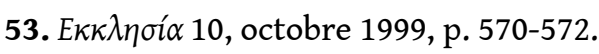

54. Cette coopération est requise par la Charte ecclésiastique de 1977, loi de l'État.

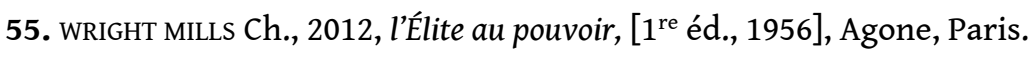




\section{RÉSUMÉS}

Dans cet article, nous examinons la place des élites religieuses en Grèce au $\mathrm{xx}^{\mathrm{e}}$ siècle, spécialement en contexte frontalier. Située aux confins de la Turquie et de la Bulgarie, restée multiconfessionnelle après 1923 , la Thrace occidentale présente au $\mathrm{xx}^{\mathrm{e}}$ siècle une configuration spécifique, contrainte - ou possible ressource - pour le clergé chrétien orthodoxe. En nous penchant sur des carrières d'évêques, en mettant l'accent sur une période de tensions intercommunautaires dans la région, nous soulignerons à quel point ces autorités «traditionnelles» s'appuient sur des modes de légitimation pluriels et entretiennent des rapports complexes avec les autres responsables régionaux. La Thrace constitue un cadre éclairant pour envisager les fonctions sociales, symboliques et parfois aux marges du politique, attendues au $\mathrm{xx}^{\mathrm{e}}$ siècle de l'Église orthodoxe et de ses dirigeants.

In this article, we examine the role of religious elites in Greece, especially in a border context. Western Thrace, near Bulgaria and Turkey, remained multidenominational after 1923 and offers a specific configuration which is a both constraint and possibly resource for the local Christian orthodox higher clergy. Focusing both on bishops' carriers and on a period of interdenominational tensions in this area, we will stress that these "traditional" authorities rely on several sources of legitimation, and maintain complex relationships with other regional leaders. The case of Thrace highlights, maybe in an acute way, the expected cultural, symbolic and even political functions of the Orthodox Church -and its leaders-, in 20th Greece's border areas.

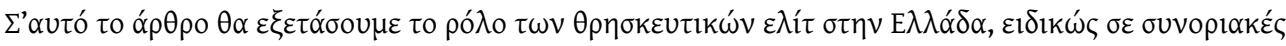

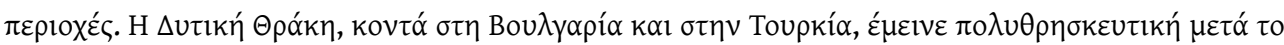

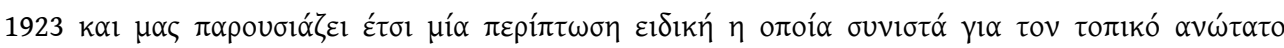

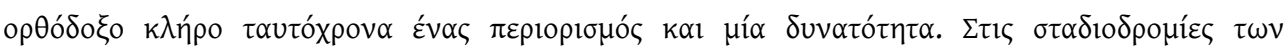

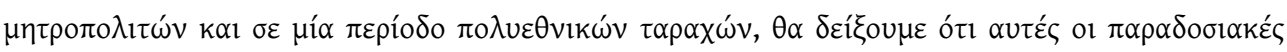

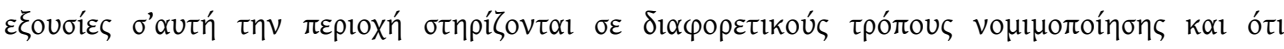

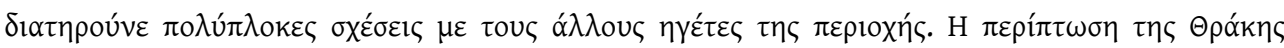

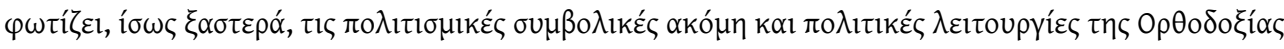

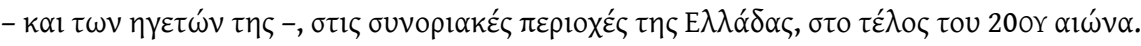

\section{INDEX}

Index géographique : Grèce, Thrace

Mots-clés : métropolites et politique, Thrace

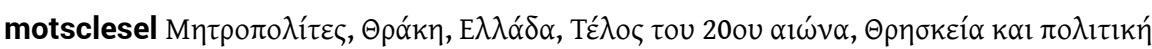

Thèmes : Religion et politique

Index chronologique : vingtième siècle -- fin

Keywords : Orthodox higher clergy, Thrace, Greece, End of the 20th century, Religion and politics 
AUTEUR

ISABELLE DÉPRET

Université libre de Bruxelles 